\title{
Influência da Obesidade Mórbida na Qualidade de Vida dos Indivíduos
}

\author{
Barros, Lívia Moreira; Moreira, Rosa Aparecida Nogueira; Frota, Natasha Marques; Araújo, \\ Thiago Moura de; Caetano, Joselany Áfio \\ Universidade Federal do Ceará — livia.moreirab@hotmail.com
}

Introdução: Diante da epidemia mundial da obesidade em todas as faixas etárias, a cirurgia bariátrica surge como um tratamento e uma possibilidade de se obter uma vida mais longa e saudável com mais qualidade, além de proporcionar novas perspectivas de vida para o indivíduo obeso. Após a cirurgia, há a minimização de problemas de ordem psicossocial e física e um aumento da auto-imagem e do bem-estar físico e psicológico que se traduzem na aquisição e participação no convívio social. Objetivo: Avaliar a Qualidade de Vida (QV) dos pacientes com obesidade mórbida do Programa de Obesidade do Estado do Ceará que são candidatos à realização da cirurgia bariátrica. Métodos: Estudo transversal realizado no período de fevereiro a junho de 2012 em um hospital referência em cirurgia bariátrica pelo Sistema Único de Saúde (SUS) no Estado do Ceará. a amostra foi composta por 64 pacientes que estão na lista de espera para realizar a cirurgia bariátrica. a coleta de dados foi feita com o questionário de Qualidade de Vida de Moorehead-Ardelt II (QoL-II). o QoL-II contém seis domínios que avaliam subjetivamente a QV do paciente: autoestima; atividade física; relações sociais; satisfação no trabalho; prazer relacionado à sexualidade e comportamento alimentar. Os dados foram tabulados no Excel e exportados para o software estatístico SPSS. Os dados relativos às variáveis sociodemográficas foram tratados a partir das frequências absolutas e relativas. para avaliar a normalidade das médias das pontuações dos domínios do QoL-II entre os pacientes, utilizou-se o teste de Kolmogorov-Smirnov e Shapiro-Wilk. o nível de significância adotado foi de $5 \%$ e o intervalo de confiança de $95 \%$. o projeto foi aprovado pelo Comitê de Ética em Pesquisa da instituição (CEP538/2011). Resultados: Observou-se que houve um predomínio do sexo feminino em que a frequência de mulheres foi de 87,5\% (56). a média de idade foi de 35,47 \pm 9,51 anos e uma variância que abrange de 20 a 57 anos. o Índice de Massa Corporal (IMC) mínimo foi de $36,1 \mathrm{~kg} / \mathrm{m} 2$ e o máximo de $68,3 \mathrm{~kg} / \mathrm{m} 2$, sendo a média de $46,4 \pm 7,09$ $\mathrm{kg} / \mathrm{m} 2$. a maioria dos indivíduos do período do pré-operatório, $57,8 \%$ (37), classificaram sua qualidade de vida como mínima e apenas 10,9\% (7) a consideraram como muito boa e $25 \%$ (16) como boa $(p<0,001)$. a média da pontuação total do questionário de qualidade de vida foi de 0,794 $\pm 1,13$ e os domínios que tiveram a menor média foram: a atividade física $(-0,420 \pm 0,35)$, o interesse sexual $(0,062 \pm 0,37)$ e o comportamento alimentar $(0,144 \pm 0,32)$. Conclusão: Diante dos resultados identificados, podemos observar que a obesidade influencia de modo negativo na qualidade de vida dos indivíduos e os domínios mais afetados estavam relacionados à alimentação, ao sedentarismo e à sexualidade, sendo, atualmente, a cirurgia bariátrica o tratamento que esses pacientes mais buscam para conseguir uma perda de peso eficaz e duradoura com, consequente, melhora na qualidade de vida.

Barros, Lívia Moreira; Moreira, Rosa Aparecida Nogueira; Frota, Natasha Marques; Araújo, Thiago Moura de; Caetano, Joselany Áfio. Influência da Obesidade Mórbida na Qualidade de Vida dos Indivíduos. In: Anais do Congresso Internacional de Humanidades \& Humanização em Saúde [= Blucher Medical Proceedings, num.2, vol.1]. São Paulo: Editora Blucher, 2014. ISSN 2357-7282

DOI 10.5151/medpro-cihhs-10826 